\title{
Decreased level of recent thymic emigrants in CD4+ and CD8+T cells from CML patients
}

\author{
Yangqiu Li*1,2, Suxia Geng1,3, Qingsong Yin1', Shaohua Chen', Lijian Yang1', Xiuli Wu1', Bo Li1, Xin Du³, \\ Christian A Schmidt 4 and Grzegorz K Przybylski*4,5
}

\begin{abstract}
Background: T-cell immunodeficiency is a common feature in cancer patients, which may relate to initiation and development of tumor. Based on our previous finding, to further characterize the immune status, T cell proliferative history was analyzed in CD4+ and CD8+ T cells from chronic myeloid leukemia (CML) patients.

Methods: Quantitative analysis of $\delta$ Rec- $\psi$ Ja signal joint T cell receptor excision circles (sjTRECs) was performed in PBMCs, CD3+, CD4+ and CD8+T cells by real-time PCR, and the analysis of 23 TRBV-D1 sjTRECs was performed by seminested PCR. Forty eight CML cases in chronic phase (CML-CP) were selected for this study and 17 healthy individuals served as controls.

Results: The levels of $\delta$ Rec- $\psi$ Ja sjTRECs in PBMCs, CD3+, CD4+, and CD8+ T cells were significantly decreased in CML patients, compared with control groups. Moreover, the numbers of detectable TRBV subfamily sjTRECs, as well as the frequency of particular TRBV-BD1 sjTRECs in patients with CML were significantly lower than those from healthy individuals.
\end{abstract}

Conclusions: We observed decreased levels of recent thymic emigrants in CD4+ and CD8+ T cells that may underlay the persistent immunodeficiency in CML patients.

\section{Background}

Chronic myeloid leukemia (CML), with the incidence of $1.5 / 100,000$ population, represents $15 \%$ of newly diagnosed leukemia cases in adults in China. The prognosis in CML improved markedly after introduction of abl tyrosine kinase inhibitors (Immatinib mesylate and its derivatives), still a lot of CML patients die due to abl mutation related drug resistance and the blast crisis [1]. Therefore further studies are needed in order to better understand the disease and to improve the patient outcome. T cell immunodeficiency was suggested to play an important role in tumor progression, facilitating the expansion of the malignant clone [2,3], although the interaction between the tumor and the immune system is still not completely understood.

\footnotetext{
*Correspondence: yangqiuli@hotmail.com, przybylg@man.poznan.pl 1 Institute of Hematology, Medical College, Jinan University, Guangzhou, 510632, China

Full list of author information is available at the end of the article
}

Most circulating mature T-cells use the $\alpha / \beta$ heterodimeric $\mathrm{T}$ cell receptor (TCR) for specific recognition of antigenic peptides in context of major histocompatibility complex (MHC) molecules. T cell differentiation in the thymus is characterized by a hierarchical order of rearrangement steps in the TCR genes, resulting in the joining of one of multiple variable $(\mathrm{V})$, diversity $(\mathrm{D})$, and joining $(J)$ gene segments. This results in each differentiating $\mathrm{T}$ cell expressing unique TCR on the surface. The $T C R$ beta locus $(T R B)$ contains at least 64 functional $\mathrm{V}$ genes $(T R B V)$ subdivided into 24 families [4]. In addition to the formation of the $\mathrm{V}(\mathrm{D}) \mathrm{J}$ coding joint, each of the $T C R$ rearrangement steps generates circular episomal DNA fragments - signal joint $\mathrm{T}$ cell recombination excision circles (sjTRECs). During the process of TCR alphadelta locus (TRAD) rearrangement, the TCR delta gene $(T R D)$, which is located within the TCR alpha gene (TRA), has to be deleted before the TRA recombination starts. Rearrangement between two TRD deleting elements, $\delta \operatorname{Rec}$ and $\psi J \alpha$, produces a $\delta R e c-\psi J \alpha$ signal joint TRECs [5-9]. sjTRECs are assumed to have a high over- 
time stability, but they can not multiply and consequently are diluted during $\mathrm{T}$ cell proliferation. A maximum of two sjTRECs can be present within one $\alpha \beta$ T cell if the corresponding rearrangement event occurs in both alleles and if the cell did not divide upon the rearrangement. sjTRECs are exported from thymus to the periphery within recent thymic emigrants (RTEs), therefore, the frequency of sjTRECs is considered to be the most accurate marker of T-cell neogenesis. Quantitative detection of sjTRECs can be applied for direct measurement of thymic output and proliferative history of $\mathrm{T}$ cells [6]. Over the last decade the technique was used to evaluate T-cell immune reconstitution in different immunodeficiency diseases [6,10-13]. To assess the proliferative history in different $T R B V$ subfamilies of T cells, quantitative analysis of $T R B V-B D$ sjTRECs has been developed [12,14,15].

The first sjTREC analysis in hematopoietic malignancy was reported by Petridou et al [16], who compared the sjTREC values in childhood B-ALL and T-ALL. Significant reduction of sjTREC values was observed in T-ALL, whereas children with B-ALL had slightly but insignificantly lower sjTRECs values compared with healthy controls. In another study, consistent with the reduction of naïve T cells, thymopoiesis (measured by sjTRECs levels) was significantly lower in 73 children with ALL than in normal controls [17]. However, little data exist regarding the proliferative history of $\mathrm{T}$ cells in myeloid leukemia patients. Recently, we published the first analysis of the sjTRECs-content in patients with acute myeloid leukemia (AML) [18]. Our previous study showed decreased $\delta$ Rec$\psi \mathrm{J} \alpha$ sjTRECs level and skewed $T R B V$ repertoire in peripheral blood mononuclear cells (PBMCs) from 20 CML cases [19]. Since the high number of CML cells in the blood might have influenced the results, in the present study, in order to more precisely characterize the immune status in chronic myeloid leukemia (CML), we analyzed both $\delta$ Rec- $\psi \mathrm{J} \alpha$ sjTRECs and $T R B V-B D$ sjTRECs in sorted CD4+ and CD8+ T cells from CML patients.

\section{Materials and methods Samples}

Forty eight newly diagnosed chronic phase CML patients, 33 males and 15 females (13-81 years old; median age: 30 years) were included in this study. BCR-ABL fusion gene was detected in all samples by RT-PCR. Seventeen healthy individuals: 6 males and 11 females (25-51 years old, median age: 28 years) served as controls. The samples were collected at Dept. of Hematology, Guangdong Province People's Hospital, all the procedures were conducted according to the guidelines of the Medical Ethics committees of the health bureau of Guangdong Province of China. sjTRECs were measured in PBMCs from all 48 cases, and CD4+ and CD8+ T cells from 19 cases. TRBV sjTRECs were determined in PBMCs, CD4+ and CD8+ T cells from 10 patients. The clinical data of the patients are listed in Table 1.

\section{Mononuclear cells isolation}

Peripheral blood mononuclear cells (PBMCs) were isolated from CML patients and healthy individuals by Ficoll-Hypaque gradient centrifugation.

\section{CD3+ cells determination}

CD3+ $\mathrm{T}$ cells percentage from PBMCs was determined by indirect immune fluorescent analysis. The PLP-fixed cytospin preparations were incubated with $200 \mu \mathrm{g} / \mathrm{ml}$ of murine anti-CD3 mAb (Boster Biological Technology Ltd, Wuhan, China), washed and incubated with 1:50 dilution of fluorescein labeled goat anti-mouse Ig (Boster Biological Technology Ltd, Wuhan, China). The slides were counterstained with Mayer's hematoxylin for 30 min. All slides were blindly evaluated using the fluorescent microscope (Nikon WFX-II, Nikon Ltd, Japan); 200 cells were counted.

\section{T cells sorting}

The CD4+ and CD8+ T cells from 19 CML cases and 17 healthy individuals were sorted using CD4 and CD8 monoclonal antibody and $\mathrm{MACS}^{\circ}$ Magnetic Cell sorting technique (Miltenyi Biotec, Bergisch Gladbach, Germany). After CD4+ and CD8+ T cells sorting, the purity was determined by indirect immune fluorescent analysis. The positive cells were around $95 \%$ to $97 \%$.

\section{DNA extraction}

Total DNA from distinct cell populations was extracted using QIAamp DNA Blood Mini Kit (QIAGEN, Germany), the quality of RNA was analyzed in $0.8 \%$ agarose gel stained with ethidium bromide and the concentration was determined by spectrophotometric analysis at 260 and $280 \mathrm{~nm}$ (Lambda 45 UV/VIS Spectrometer, Perkin Elmer USA).

\section{Real-time quantitative PCR (RQ-PCR)}

Quantitative detection of $\delta R e c-\psi J \alpha$ sjTRECs in DNA from PBMCs and sorted CD4+ or CD8+ T cells was preformed by real-time PCR using the ABI PRISM 7700 Sequence Detector TaqMan (PE Biosystems, Foster City, CA). PCR was performed as described by previous studies $[15,20]$. To precisely determine the percentage of cells carrying sjTREC we constructed a duplex vector including a fragment of the $\delta$ Rec- $\psi J \alpha$ (sjTREC) and a fragment of the RAG2 gene used as a reference. The RAG2 was cloned first in the T-A acceptor site and subsequently the sjTREC was cloned in to the EcoRV restriction site of the TOPO TA Vector (Invitrogen, Groning, The Netherlands). Based on the DNA concentration, measured by spectrophotometry and confirmed by a quantitative gel eletrophoresis, standard dilutions of the vector from $10^{7}$ 
Table 1: Clinical data of CML patients

\begin{tabular}{|c|c|c|c|c|c|c|c|}
\hline No. & sex & age & $\begin{array}{l}\text { WBC } \\
\left(\times 10^{9} / \mathrm{L}\right)\end{array}$ & $\begin{array}{l}\text { Blast+pro } \\
\text { myelocyte } \\
\text { cells (\%) }\end{array}$ & $\begin{array}{l}\text { Platelets } \\
\left(\times 10^{9} / \mathrm{L}\right)\end{array}$ & CD3+\% & $\begin{array}{l}\text { CD4+/ } \\
\text { CD8+ } \\
\text { cells sorted }\end{array}$ \\
\hline $\mathrm{C} 1$ & $\mathrm{~F}$ & 49 & 213.59 & 9 & 147 & 28.91 & Yes \\
\hline $\mathrm{C} 2$ & M & 16 & 351.16 & 0 & 345 & 4.71 & Yes \\
\hline $\mathrm{C} 3$ & M & 58 & 59.93 & 6 & 144 & 11.01 & Yes \\
\hline $\mathrm{C} 4$ & M & 20 & 124 & 0 & 605 & 18.2 & Yes \\
\hline $\mathrm{C} 5$ & M & 25 & 256.82 & 6 & 109 & 13.8 & Yes \\
\hline $\mathrm{C} 6$ & M & 15 & 333.95 & 8.5 & 208 & 10.8 & Yes \\
\hline $\mathrm{C} 7$ & $\mathrm{~F}$ & 31 & 294.91 & 3 & 252 & 10.46 & Yes \\
\hline $\mathrm{C} 8$ & M & 30 & 118.55 & 5 & 440 & 9.6 & Yes \\
\hline $\mathrm{C} 9$ & M & 24 & 244.05 & 9 & 750 & 12.04 & Yes \\
\hline C10 & M & 61 & 279 & 10 & 993 & 11.8 & Yes \\
\hline $\mathrm{C} 11$ & $\mathrm{~F}$ & 30 & 99.8 & 6.5 & 378 & 2.1 & Yes \\
\hline $\mathrm{C} 12$ & M & 38 & 103.66 & 1 & 181 & 10.4 & Yes \\
\hline C13 & $\mathrm{F}$ & 20 & 450.45 & 1 & 396 & 12.1 & Yes \\
\hline C14 & M & 42 & 81.6 & 6 & 85 & 14.18 & Yes \\
\hline $\mathrm{C} 15$ & M & 73 & 196 & 8 & 1531 & 9.1 & Yes \\
\hline C16 & M & 31 & 129 & 3.5 & 285 & 28.4 & Yes \\
\hline C17 & M & 22 & 76.6 & 5.5 & 171 & 19.64 & Yes \\
\hline C18 & M & 20 & 112.7 & 5 & 596 & 42.5 & Yes \\
\hline C19 & $\mathrm{F}$ & 19 & 7 & 4 & 125 & 28.0 & Yes \\
\hline $\mathrm{C} 20$ & M & 61 & 44.9 & 12 & 77 & 13.6 & No \\
\hline $\mathrm{C} 21$ & $\mathrm{~F}$ & 13 & 314.78 & 3 & 640 & 32 & No \\
\hline $\mathrm{C} 22$ & $M$ & 59 & 18.54 & 2 & 695 & 56.89 & No \\
\hline $\mathrm{C} 23$ & $M$ & 50 & 31.5 & 0 & 163 & 36.51 & No \\
\hline $\mathrm{C} 24$ & $M$ & 35 & 5.1 & 5 & 283 & 38.6 & No \\
\hline $\mathrm{C} 25$ & $\mathrm{~F}$ & 66 & 62.87 & 2 & 657 & 7.8 & No \\
\hline $\mathrm{C} 26$ & $\mathrm{~F}$ & 30 & 160 & 16 & 842 & 12.4 & No \\
\hline $\mathrm{C} 27$ & $\mathrm{~F}$ & 26 & 114.17 & 2 & 222 & 19.1 & No \\
\hline $\mathrm{C} 28$ & $M$ & 26 & 5.3 & 0 & 118 & 44.7 & No \\
\hline $\mathrm{C} 29$ & $M$ & 15 & 185.9 & 3 & 291 & 10.5 & No \\
\hline C30 & $M$ & 27 & 101.5 & 3 & 326 & 42.5 & No \\
\hline C31 & $F$ & 21 & 29.7 & 2 & 296 & 26.67 & No \\
\hline C32 & $M$ & 22 & 111.92 & 0 & 115 & 9.17 & No \\
\hline C33 & $F$ & 75 & 267 & 7 & 258 & 11.2 & No \\
\hline C34 & $M$ & 29 & 0.08 & 2 & 34 & 18.75 & No \\
\hline C35 & $M$ & 26 & 61.67 & 9 & 661 & 31.5 & No \\
\hline C36 & $M$ & 43 & 170 & 0 & 671 & 40.66 & No \\
\hline C37 & $M$ & 36 & 43.87 & 6 & 69 & 32.07 & No \\
\hline C38 & $M$ & 38 & 58.55 & 0 & 3363 & 18.95 & No \\
\hline C39 & $M$ & 29 & 132.4 & 10.5 & 1221 & 26.9 & No \\
\hline $\mathrm{C} 40$ & $\mathrm{~F}$ & 55 & 130.21 & 4 & 204 & 14.2 & No \\
\hline C41 & $M$ & 44 & 485.1 & 1 & 514 & 27.2 & No \\
\hline C42 & $F$ & 16 & 1.39 & 0 & 46 & 38.2 & No \\
\hline
\end{tabular}


Table 1: Clinical data of CML patients (Continued)

\begin{tabular}{llllllll}
\hline C43 & F & 35 & 102.85 & 1 & 335 & 41.7 & No \\
C44 & F & 25 & 33.44 & 6 & 470 & 35.0 & No \\
C45 & M & 81 & 30.3 & 0 & 747 & 9.02 & No \\
C46 & $M$ & 38 & 154 & 1 & 485 & 13.75 & No \\
C47 & M & 30 & 2.32 & 0 & 46 & 41.9 & No \\
C48 & M & 25 & 7.63 & 11 & 139 & 49.3 & No \\
\hline
\end{tabular}

to $10^{1}$ copies were prepared [15,20]. In brief, PCR of $25 \mu \mathrm{l}$ total volume was performed with approximately $100 \mathrm{ng}$ of genomic DNA, 25 pmol of each primers (TREC-1 and TREC-2 for sjTRECs, RAG2-for and RAG2-back for RGA2 amplification), $10 \mathrm{nmol}$ each dNTP, $1.5 \mathrm{U}$ AmpliTaq Gold (Applied Biosystems, Branchburg, New Jersey, USA), 5 pmol of 6FAM-TAMRA probe and PCR Buffer including $4.5 \mathrm{mM} \mathrm{MgCl}$. After the initial denaturation at $95^{\circ} \mathrm{C}$ for $5 \mathrm{~min}, 45$ cycles consisting of $95^{\circ} \mathrm{C}$ for $30 \mathrm{sec}$ and $67^{\circ} \mathrm{C}$ for $1 \mathrm{~min}$ were performed. If no TRECs were detected in a sample, PCR was repeated with more DNA.

\section{TRBV-BD1 sjTRECs detection by semi-nest PCR}

Twenty three $T R B V-B D 1$ sjTRECs were amplified by semi-nest PCR from different amounts of genomic DNA $\left(1.3 \mu \mathrm{g}, 325 \mathrm{ng}\right.$ or $65 \mathrm{ng}$, corresponding to $2 \times 10^{5}, 5 \times 10^{4}$ or $1 \times 10^{4}$ cells respectively) isolated from PBMCs, CD4+ and CD8+ T cells. Two nested 5' TRBD1 primers, located upstream of the segment, and twenty three 3' TRBV primers ( $B V 1-19$ and $B V 21-24)$ were used [15,20]. Since the $T R B V 20-B D 1$ rearrangement occurs by inversion, it does not generate a sjTREC. In the first round PCR, $2 \mu \mathrm{l}$ of genomic DNA were amplified in a $10 \mu$ reaction mixture containing: $0.375 \mu \mathrm{M}$ external sense and antisense primers, $0.1 \mathrm{mM}$ dNTP, $1.5 \mathrm{mM} \mathrm{MgCl}_{2}, 1 \times$ PCR buffer and $1 \mathrm{U}$ Taq polymerase (GoTaq ${ }^{\circ}$ Flexi DNA polymerase, Promega, Madison, WI, USA) using the DNA thermal cycler. After 3 min denaturation at $94^{\circ} \mathrm{C}, 30$ PCR cycles were performed, each cycle consisting of $94^{\circ} \mathrm{C}$ for 1.5 $\min , 65^{\circ} \mathrm{C}$ for $1 \mathrm{~min}$ and $72^{\circ} \mathrm{C}$ for $1 \mathrm{~min}$, and a final $6 \mathrm{~min}$ elongation at $72^{\circ} \mathrm{C}$. Then, the products were stored at $4^{\circ} \mathrm{C}$. In the second round PCR, 25 cycles of amplification were carried out with $2 \mu \mathrm{l}$ of the first PCR products, the same BV primer and the internal sense BD1 primer.

\section{Statistical analysis}

Univariate analyses were done using the Mann-Whitney test to compare the numbers of $\delta$ Rec- $\psi J \alpha$ sjTRECs and detectable TRBV-BD1 sjTRECs in CML and healthy control groups. The chi square test was used to compare the frequency of TRBV-BD1 sjTRECs in PBMCs in CML and healthy control groups. Pearson correlation and linear regression analysis were used to estimate the correlation between age and sjTRECs numbers.

\section{Results}

Decreased level of $\delta$ Rec- $\psi J a$ sjTRECs in PBMCs, CD4+ and CD8+ cells from CML patients

The absolute numbers of sjTRECs and RAG2 were measured in two independent assays and sjTREC content per 1000 PBMCs was calculated using a formula $\mathrm{n}=2 \times 1000$ $\times[\operatorname{sjTREC}(1)+\operatorname{sjTREC}(2)] /[\operatorname{RAG} 2(1)+\operatorname{RAG} 2(2)][15]$. The absolute numbers of sjTRECs in T cells were determined by the percentage of CD3-positive cells $(n=$ sjTRECs/ $1000 \mathrm{PBMCs} \div \mathrm{CD} 3 \%$ ). The CD3+ percentage in PBMCs from healthy individuals was $62.32 \pm 4.72 \%$, and $22.89 \pm$ $13.76 \%$ in PBMCs from CML patients. The sjTRECs levels in PBMCs, CD3+, CD4+ and CD8+ $\mathrm{T}$ cells from patients with CML are shown in Figure 1. In comparison with the sjTRECs in healthy individuals $(3.76 \pm 3.42$ copies/1000 PBMCs, $5.87 \pm 4.96$ copies/1000 CD3+ cells, $5.62 \pm 6.45$ copies $/ 1000$ CD $4+T$ cells, $6.79 \pm 7.1$ copies/ 1000 CD8+T cells), a dramatic reduction of sjTRECs values was found in patients with CML $(0.23 \pm 0.38$ copies/

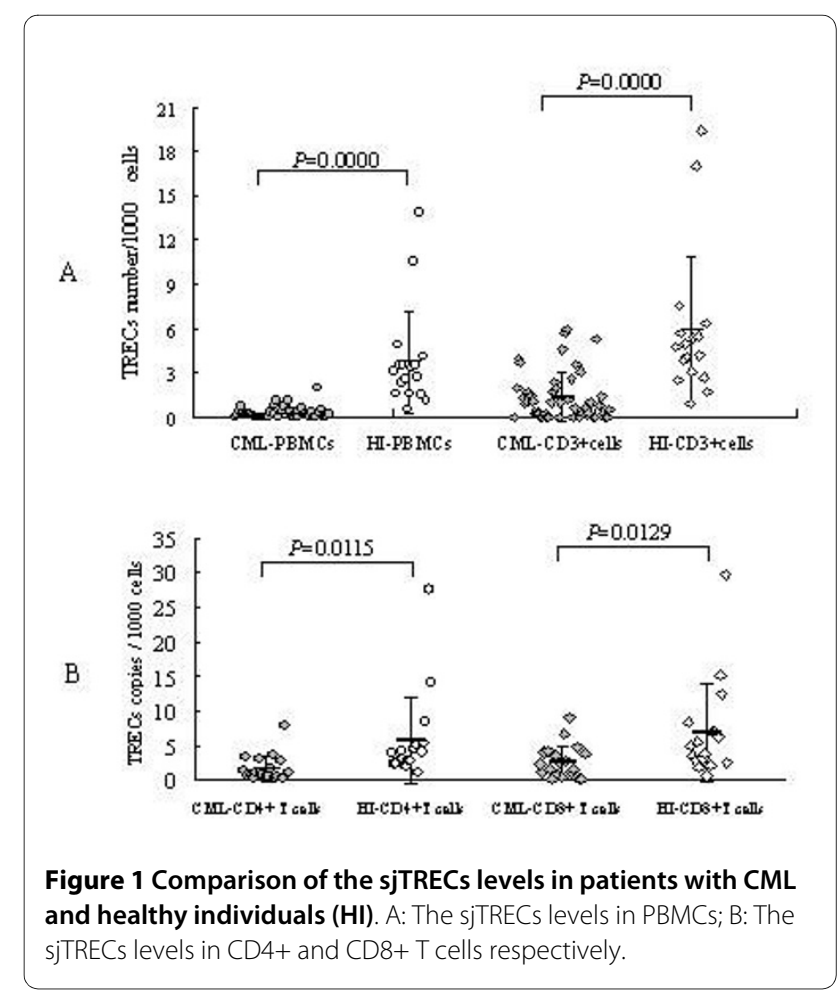


1000 PBMCs, $1.34 \pm 1.63$ copies/1000 CD3+ cells, $1.49 \pm$ $1.88 / 1000 \mathrm{CD} 4+\mathrm{T}$ cells, $2.52 \pm 2.43$ copies/1000 CD8 $+\mathrm{T}$ cells) $(\mathrm{p}<0.0001, \mathrm{p}<0.0001, \mathrm{p}=0.0115$ and $\mathrm{p}=0.0129$, respectively).

The numbers of sjTRECs in PBMCs and sorted T cells from CML were higher in females than in males. The values were: the PBMCs group: $0.19 \pm 0.25$ copies/1000cells in male $(n=33)$ versus $0.43 \pm 0.56$ copies $/ 1000$ cells in female $(\mathrm{n}=15)(\mathrm{p}=0.0467)$, in the CD3+T cells group: $1.05 \pm 1.21$ copies/1000cells in male $(n=33)$ versus $1.97 \pm$ 2.25 copies $/ 1000$ cells in female $(\mathrm{n}=15)(\mathrm{p}=0.0712)$, in the CD4+T cells group: $1.4 \pm 2.08$ copies/1000cells in male $(\mathrm{n}=14)$ versus $1.74 \pm 1.31$ copies/1000cells in female $(\mathrm{n}=5)(\mathrm{p}=0.739)$, and in the CD8+T cells group: $1.66 \pm 1.63$ copies $/ 1000$ cells in male $(n=14)$ versus $4.95 \pm$ 2.82 copies $/ 1000$ cells in female $(n=5)(p=0.0053)$. Similar results were found in healthy individual group (data not shown). Although the differences between genders were quite obvious, they were not statistically significant, except for PBMCs and CD8+ cells in CML patients.

\section{Lower frequencies of 23 TRBV-BD1 sjTRECs in PBMCs, CD4+ and CD8+ cells from CML patients}

The TRBV-BD1 sjTRECs from TRBV1-19 and TRBV2124 were analyzed by semi-nested PCR, using different amounts of DNA (corresponding to $2 \times 10^{5}, 5 \times 10^{4}$ or $1 \times$ $10^{4}$ cells respectively). Samples were amplified to estimate the frequency of TCR TRBV-BD1 sjTRECs and the sequences of the junction regions of each $T R B V-B D 1$ sjTRECs were confirmed by PCR products direct sequencing (data not shown).

The number of detectable $T R B V$ subfamily sjTRECs differed significantly between CML and healthy control in $2 \times 10^{5}, 5 \times 10^{4}$ and $1 \times 10^{4}$ PBMCs or in $1 \times 10^{4}$ of CD4+ and CD8+ T cells (Figure 2). Comparison of the frequencies of $23 T R B V-B D 1$ sjTRECs in PBMCs

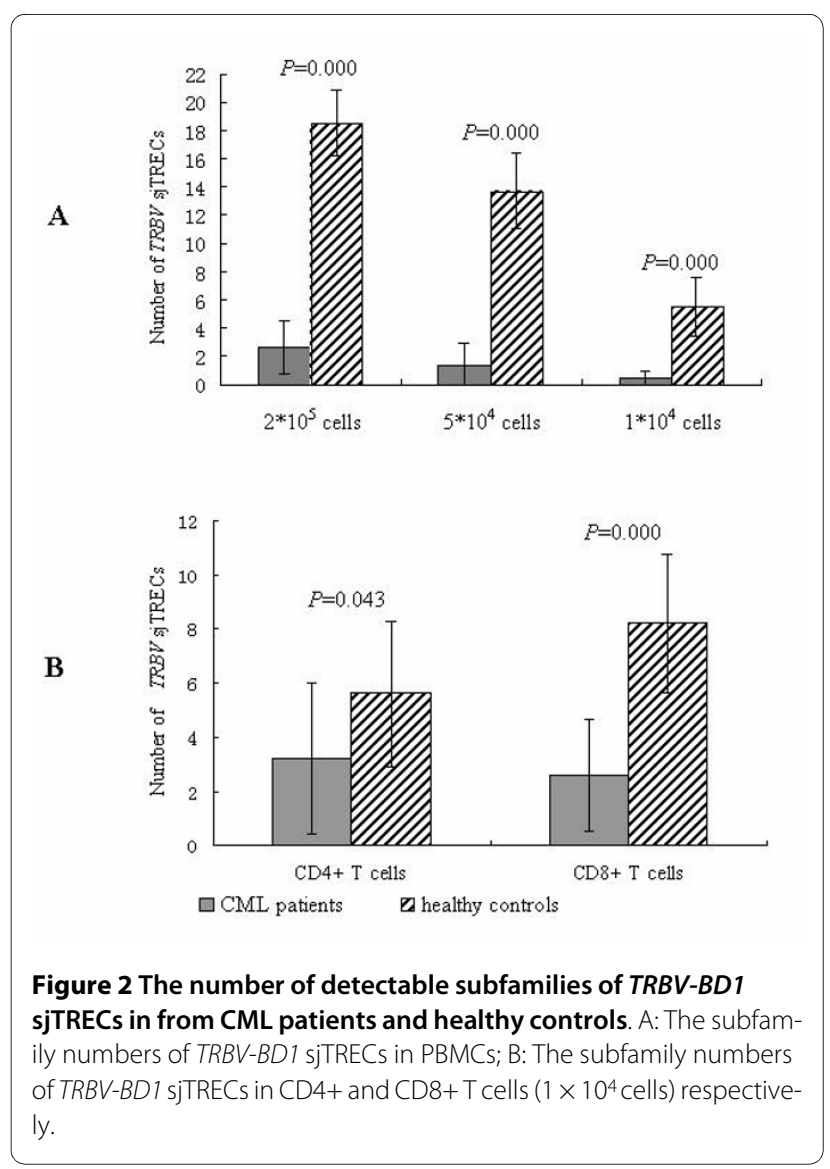

between CML patients and normal controls at different amounts of DNA level showed that the frequencies of the most $T R B V$ subfamily sjTRECs were significantly lower than those from healthy individuals, especially at the higher cellular concentration $\left(2 \times 10^{5}\right.$ PBMCs) (Figure 3$)$. But the significant difference was found only in few subfamilies ( $B V 2, B V 10, B V 12$ and $B V 14$ in CD8+T cells) when comparing the frequency of $T R B V$ subfamily

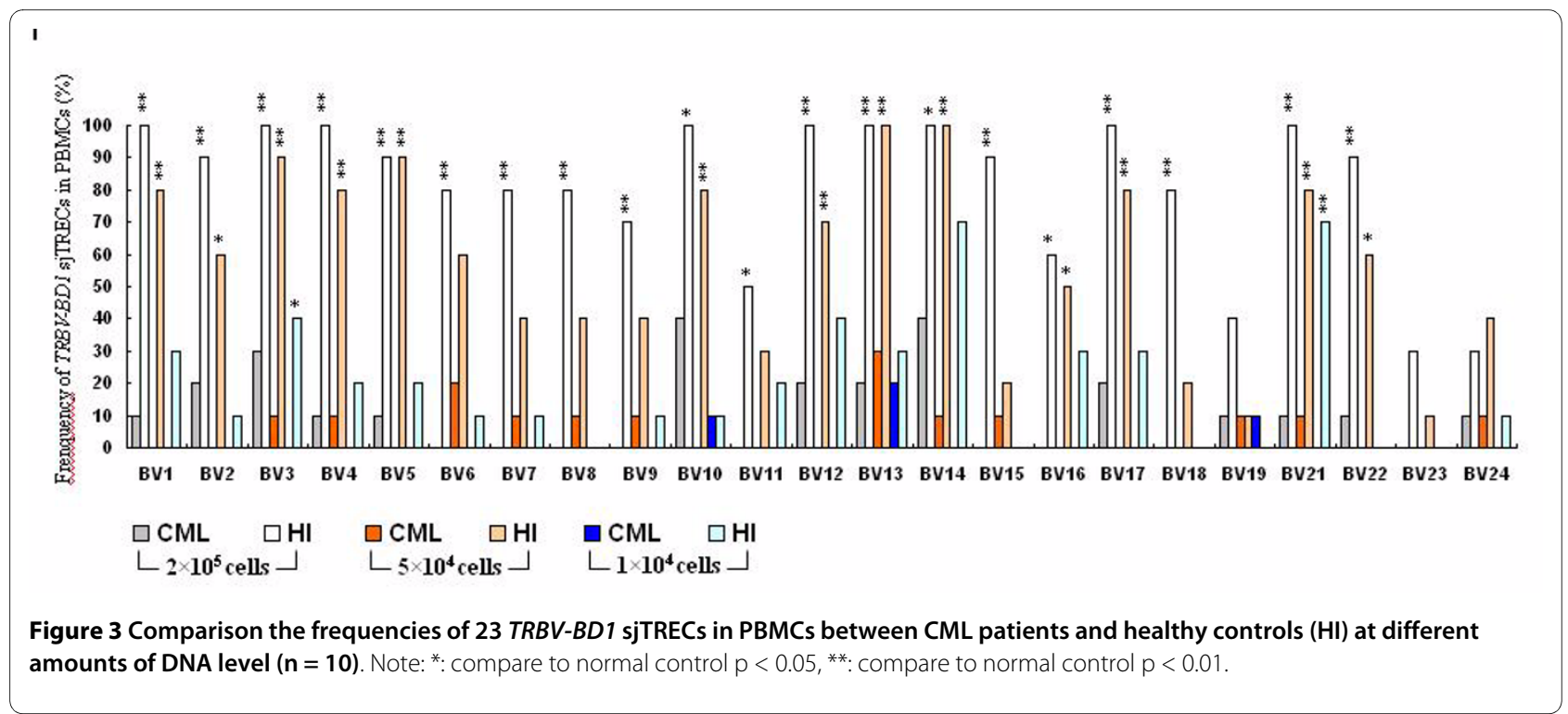




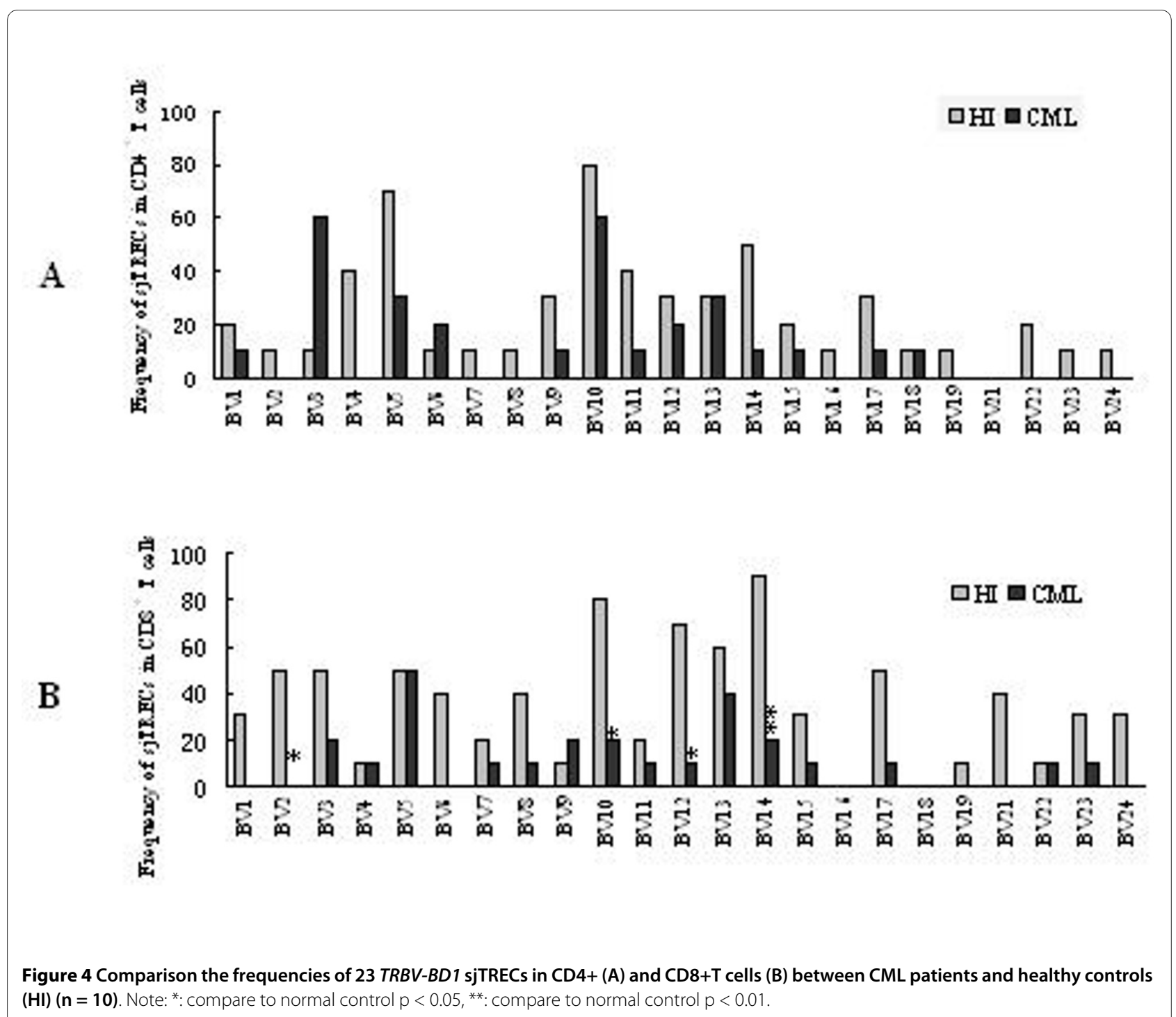

sjTRECs in CD4+ and CD8+ T cells at $1 \times 10^{4}$ concentration between both group (Figure 4).

\section{Discussion}

In patients with CML, cellular immune deficiency is a common feature which may be due to decreased output of recent thymic emigrants, the abnormal expression of $\mathrm{T}$ cell receptor repertoire and, may in part, due to altered expression of TCR-CD3 complex. Our previous study showed decreased $\delta$ Rec- $\psi \mathrm{J} \alpha$ sjTRECs level and skewed $T R B V$ repertoire in peripheral blood mononuclear cells (PBMCs) from CML patients [19,21]. And TCR $\zeta$ chain expression was decreased in $\mathrm{T}$ cells from patients with CML [22,23].

In order to further evaluate the T-cell immune function, the $\mathrm{T}$ cell proliferative history in CML patients was analyzed. The sjTRECs-content in PBMCs and CD3+ T cells from $48 \mathrm{CML}$ cases was determined. The results confirmed our previous smaller study [19]. We showed a dramatic reduction of sjTRECs values in CML patients. In some cases no sjTRECs could be detected in $40000 \mathrm{~T}$ cells. This suggests poor thymic output in CML patients, which may be even more pronounced than in ALL patients [16]. To date there are only a few papers describing TRECs level in hematopoietic malignancies [16,17]. The exact value of sjTRECs level in PBMCs from CML patients are influenced by contaminating normal non- $\mathrm{T}$ cells and leukemia blast cells; therefore the sjTRECs numbers were normalized with the percentage of CD3+cells in the analyzed samples. Furthermore, we analyzed sjTRECs in sorted CD4+ and CD8+ T cells. This is the most sensitive and accurate method for quantitation of naïve T-cells. It allows also the comparison of sjTRECs levels in CD4+ and CD8+ subsets. The levels of sjTRECsexpressing CD4+ and CD8+ T cells were significantly decreased in CML patients, as compared with age and sex 
matched healthy individuals. The decrease of sjTRECs levels was similar in both $\mathrm{T}$ cell subsets. These findings suggest that an impaired thymic output function and, as a consequence, an altered ability to maintain $\mathrm{T}$ cell homeostasis, which may play an important role in the immunodeficiency in CML patients. However, whether this is due to the clonal expansion of T-cells to antigens, for example leukemia associated antigens, or reflects the impairment of immune function associated with the malignancy, remains an open question [7,24-27].

Pido-Lopez et al showed that the decline in number of recent thymic emigrants in the blood with increasing age is gender-linked [28]. Peripheral blood from female contained significantly higher levels of sjTRECs per CD3+ T cell than blood from males. Also in children, the number of sjTRECs was higher in healthy girls than in healthy boys, and a similar pattern was evident in T-cell malignancies [16]. In the present study, we observed slightly, but in-significantly higher sjTRECs levels in healthy females, however, the number of sjTRECs was statistically higher in PBMCs and CD8+ $\mathrm{T}$ cells from female CML patients.

The majority of studies published previously focused only on the total thymic output, as measured by quantitative analysis of $\delta$ Rec- $\psi J \alpha$ sjTRECs [6]. This approach doesn't allow the evaluation of the complexity of thymic output in different TRBV subfamily naïve T cells, which is an important factor in immune competence. In this study, we analyzed the total 23 subfamilies of TRBV-DB1 sjTRECs in PBMCs, CD4+ and CD8+ T-cells from CML patients by a semi-nested PCR. The results indicate that the percentage of cases positive for TRBV-DB1 sjTRECs varies in different $B V$ subfamilies in healthy controls; the highest for TRBV1, 3, 4, 10, 12-14, 17 and $V 21$, which could be detected in all 10 samples (at $2 \times 10^{5}$ PBMCs). The most important observation in this study was the significantly lower frequency of $23 T R B V-B D 1$ sjTRECs in PBMCs, as well as in CD4+ and CD8+ T cells from CML patients as compared with healthy individuals, indicating poor thymic output in CML patients. The results further support and explain the significant reduction of recent thymic emigrant numbers in peripheral blood of CML patients, as measured by quantitative detection of $\delta$ Rec$\psi J \alpha$ sjTRECs.

In conclusion, this is, to our knowledge, the first characterization of thymic output function in $\mathrm{CD} 4+$ and CD8+ T cells from CML patients based on analyses of both $\delta$ Rec- $\psi$ J $\alpha$ sjTRECs and TRBV-DB1 subfamily specific sjTRECs. We showed a prominent decrease of sjTRECs levels in CML, indicating the reduction of recent thymic emigrants affects the majority of TRBV subfamilies.
Competing interests

The authors declare that they have no competing interests.

\section{Authors' contributions}

YQL, CAS and GKP were responsible for study design and data management. SXG and SHC performed the real-time PCR, QSY and LJY performed the seminested PCR, XLW and BL performed the statistical analysis, XD collected samples. All authors read and approved the final manuscript.

\section{Acknowledgements}

The authors thank Prof. Dr. John Yeuk-Hon Chan for critical reading of this manuscript. The study was sponsored by grants from National Natural Science Foundation of China (No. 30270579) and Natural Science Foundation of Guangdong Province (No.23001, 9251063201000001).

\section{Author Details}

${ }^{1}$ Institute of Hematology, Medical College, Jinan University, Guangzhou, 510632, China, 2Key Laboratory for Regenerative Medicine of Ministry of Education, Jinan University, Guangzhou, 510632, China, ${ }^{3}$ Department of Hematology, Guangdong Province People's Hospital, Guangzhou 510080 China, ${ }^{4}$ Department of Hematology and Oncology, Ernst-Moritz-Arndt University Greifswald, Greifswald 17487, Germany and 5 Institute of Human Genetics, Polish Academy of Sciences, Poznan, Poland

Received: 28 November 2009 Accepted: 14 May 2010

Published: 14 May 2010

\section{References}

1. Jabbour E, Cortes J, Kantarjian H: Treatment selection after imatinib resistance in chronic myeloid leukemia. Target Oncol 2009, 4:3-10.

2. Hadden JW: Immunodeficiency and cancer: prospects for correction. Int Immunopharmacol 2003, 3:1061-71.

3. Costello RT, Rey J, Fauriat C, Gastaut JA, Olive D: New approaches in the immunotherapy of haematological malignancies. Eur J Haematol 2003, 70:333-45.

4. Pannetier C, Even J, Kourilsky P: T-cell repertoire diversity and clonal expansions in normal and clinical samples. Immunol Today 1995, 16:176-81.

5. De Villartay JP, Hockett RD, Coran D, Korsmeyer SJ, Cohen DI: Deletion of the human T-cell receptor $\delta$-gene by a site specific recombination. Nature 1988, 335:170-4.

6. Douek DC, McFarland RD, Keiser PH, Gage EA, Massey JM, Haynes BF, Polis MA, Haase AT, Feinberg MB, Sullivan JL, Jamieson BD, Zack JA, Picker LJ, Koup RA: Changes in thymic function with age and during the treatment of HIV infection. Nature 1998, 396:690-5.

7. Hazenberg MD, Verschuren MC, Hamann D, Miedema F, van Dongen JJ: T cell receptor excision circles as markers for recent thymic emigrants: basic aspects, technical approach, and guidelines for interpretation. J Mol Med 2001, 79:631-40.

8. Geenen V, Poulin JF, Dion ML, Martens H, Castermans E, Hansenne I, Moutschen M, Sekaly RP, Cheynier R: Quantification of T cell receptor rearrangement excision circles to estimate thymic function: an important new tool for endocrine-immune physiology. J Endocrinol 2003, 176:305-11.

9. Al-Harthi L, Marchett G, Steffens CM, Poulin J, Sekaly R, Landay A: Detection of T cell receptor circles (TRECs) as biomarkers for de novo $T$ cell synthesis using a quantitative polymerase chain reaction-enzyme linked immunosorbent assay (PCR-ELISA). J Immunol Methods 2000, 237:187-97

10. Douek D, Vescio RA, Betts MR, Brenchley JM, Hill BJ, Zhang L, Berenson JR, Collins RH, Koup RA: Assessment of thymic output in adults after haematopoietic stem-cell transplantation and prediction of T-cell reconstitution. Lancet 2000, 355:1875-81.

11. Svaldi M, Lanthaler AJ, Dugas M, Lohse P, Pescosta N, Straka C, Mitterer M: T-cell receptor excision circles: a novel prognostic parameter for the outcome of transplantation in multiple myeloma patients. $\mathrm{Br} J$ Haematol 2003, 122:795-801.

12. Poulin JF, Sylvestre M, Champagne P, Dion ML, Kettaf N, Dumont A, Lainesse M, Fontaine P, Roy DC, Perreault C, Sékaly RP, Cheynier R: Evidence for adequate thymic function but impaired naive T-cell survival following allogeneic hematopoietic stem cell transplantation 
in the absence of chronic graft-versus-host disease. Blood 2003, 102:4600-7.

13. Przybylski GK, Kreuzer KA, Siegert W, Schmidt CA: No recovery of T-cell receptor excision circles (TRECs) after non-myeloablative allogeneic hematopoietic stem cell transplantation is correlated with the onset of GvHD. J Appl Genet 2007, 48:397-404.

14. Poulin JF, Viswanathan MN, Harris JM, Komanduri KV, Wieder E, Ringuette $\mathrm{N}$, Jenkins M, McCune JM, Sékaly RP: Direct evidence for thymic function in adult humans. JExp Med 1999, 190:479-86.

15. Li Y, Chen S, Yang L, Yin Q, Geng S, Wu X, Schmidt CA, Przybylski GK: TRAV and $T R B V$ repertoire, clonality and the proliferative history of umbilical cord blood T cells. Transplant Immunol 2007, 18:151-8.

16. Petridou E, Klimentopoulou AE, Moustaki M, Kostrikis LG, Hatzakis A, Trichopoulos D: Recent thymic emigrants and prognosis in T- and B-cell childhood hematopoietic malignancies. Int J Cancer 2002, 101:74-7.

17. Haining WN, Evans J, Seth N, Callaway G, Wucherpfennig K, Nadler L, Guinan E: Rapid T Cell Response to Vaccination Can Occur without Antibody Response in Children Post HSCT. Blood 2004, 104(11):614a.

18. Li Y, Yin Q, Yang L, Chen S, Geng S, Wu X, Zhong L, Schmidt CA, Przybylski GK: Reduced levels of recent thymic emigrants in acute myeloid leukemia patients. Cancer Immunol Immunother 2009, 58:1047-55.

19. Geng SX, Li YQ, Chen SH, Yang LJ, Yin QS, Wu XL, Zhang XL: Peripheral blood naive $T$ cell level and its $T$ cell receptor Vbeta repertoire usage profile in patients with chronic myelogenous leukemia. Zhonghua Xue YeXue Za Zhi 2005, 26:413-6. (Chinese)

20. Kimmig S, Przybylski GK, Schmidt CA, Laurisch K, Mowes B, Radbruch A, Thiel A: Two subsets of naive $T$ helper cells with distinct $T$ cell receptor excision circle content in human adult peripheral blood. J Exp Med 2002, 195:789-94.

21. Wang L, Zhu K, Zha X, Chen S, Yang L, Chen S, Li Y: Evolution of T-cell clonality in a patient with $\mathrm{Ph}$-negative acute lymphocytic leukemia occurring after interferon and imatinib therapy for Ph-positive chronic myeloid leukemia. J Hematol Oncol 2010, 3:14

22. Rossi E, Matutes E, Morilla R, et al:: Zeta chain and CD28 are poorly expressed on T lymphocytes from chronic lymphocytic leukaemia. Leukemia 1996, 10:494-7.

23. Chen $S$, Yang $L$, Chen $S$, Li Y: TCR $\zeta$ chain expression in T cells from patients with CML. Hematology 2009, 14:95-100

24. Farace F, Orlanducci F, Dietrich PY, Gaudin C, Angevin E, Courtier MH, Bayle C, Hercend T, Triebel F: T cell repertoire in patients with $B$ chronic lymphocytic leukemia. Evidence for multiple in vivo T cell clonal expansions. J Immunol 1994, 153:4281-90

25. Serrano D, Monteiro J, Allen SL, Kolitz J, Schulman P, Lichtman SM, Buchbinder A, Vinciguerra VP, Chiorazzi N, Gregersen PK: Clonal expansion within the CD4+CD57+ and CD8+CD57+ T cell subsets in chronic lymphocytic leukemia. J Immuno 1997, 158:1482-9.

26. Alatrakchi N, Farace F, Frau E, Carde P, Munck JN, Triebel F: T-cell clonal expansion in patients with B-cell lymphoproliferative disorders. $J$ Immunother 1998, 21:363-70.

27. Li Y: Recent thymic output function in patients with hematological malignancy. Hematology 2005, 10:297-305.

28. Pido-Lopez J, Imami N, Aspinall R: Both age and gender affect thymic output: more recent thymic migrants in females than males as they age. Clin Exp Immunol 2001, 125:409-13.

Submit your next manuscript to BioMed Centra and take full advantage of:

- Convenient online submission

- Thorough peer review

- No space constraints or color figure charges

- Immediate publication on acceptance

- Inclusion in PubMed, CAS, Scopus and Google Scholar

- Research which is freely available for redistribution

Submit your manuscript at www.biomedcentral.com/submit
C Biomed Central 